\title{
Strong Coupling Constant from Scaling Violations in Fragmentation Functions
}

\author{
Bernd A. Kniehl, ${ }^{1}$ Gustav Kramer, ${ }^{1}$ and Björn Pötter ${ }^{2}$ \\ ${ }^{1}$ II. Institut für Theoretische Physik, Universität Hamburg, Luruper Chaussee 149, 22761 Hamburg, Germany \\ ${ }^{2}$ Max-Planck-Institut für Physik (Werner-Heisenberg-Institut), Föhringer Ring 6, 80805 Munich, Germany
}

(Received 30 March 2000)

\begin{abstract}
We present a new determination of the strong coupling constant $\alpha_{s}$ through the scaling violations in the fragmentation functions for charged pions, charged kaons, and protons. In our fit we include the latest $e^{+} e^{-}$annihilation data from CERN LEP1 and SLAC SLC on the Z-boson resonance and older, yet very precise, data from SLAC PEP at center-of-mass energy $\sqrt{s}=29 \mathrm{GeV}$. At next-to-leading order, we find $\alpha_{s}^{(5)}\left(M_{Z}\right)=0.1170 \pm 0.0073$. A new world average of $\alpha_{s}$ is given.
\end{abstract}

PACS numbers: 13.65.+i, 13.85.Ni, 13.87.Fh

The strong force acting between hadrons is one of the four fundamental forces of nature. It is now commonly believed that the strong interactions are correctly described by quantum chromodynamics (QCD), the SU(3) gauge field theory which contains colored quarks and gluons as elementary particles. The strong coupling constant $\alpha_{s}^{\left(n_{f}\right)}(\mu)=g_{s}^{2} /(4 \pi)$, where $g_{s}$ is the QCD gauge coupling, is a basic parameter of the standard model of elementary particle physics; its value $\alpha_{s}^{(5)}\left(M_{Z}\right)$ at the $Z$-boson mass scale is listed among the constants of nature in the Review of Particle Physics [1]. Here $\mu$ is the renormalization scale, and $n_{f}$ is the number of active quark flavors, with mass $m_{q} \ll \mu$. The formulation of $\alpha_{s}^{\left(n_{f}\right)}(\mu)$ in the modified minimal-subtraction ( $\overline{\mathrm{MS}}$ ) scheme, with four-loop evolution [2] and three-loop matching at the flavor thresholds [3], is described in Ref. [3].

There are a number of processes in which $\alpha_{s}^{(5)}\left(M_{Z}\right)$ can be measured (see Refs. [1,4] for recent reviews). A reliable method to determine $\alpha_{s}^{(5)}\left(M_{Z}\right)$ is through the extraction of the fragmentation functions (FF's) in the annihilation process

$$
e^{+} e^{-} \rightarrow(\gamma, Z) \rightarrow h+X,
$$

which describes the inclusive production of a single charged hadron, $h$. Here $h$ may refer either to a specific charged-hadron species, such as $\pi^{ \pm}, K^{ \pm}$, or $p / \bar{p}$, or to the sum of all charged hadrons. The partonic cross sections pertinent to process (1) can be entirely calculated in perturbative QCD with no additional input, except for $\alpha_{s}$. They are known at next-to-leading order (NLO) [5] and even at next-to-next-to-leading order [6]. The subsequent transition of the partons into hadrons takes place at an energy scale of the order of $1 \mathrm{GeV}$ and can, therefore, not be treated in perturbation theory. Instead, the hadronization of the partons is described by FF's $D_{a}^{h}\left(x, Q^{2}\right)$. Their values correspond to the probability that the parton $a$, which is produced at short distance, of order $1 / Q$, fragments into the hadron $h$ carrying the fraction $x$ of the momentum of $a$. In the case of process (1), $Q$ is typically of the order of the center-of-mass $(\mathrm{CM})$ energy $\sqrt{s}$. Given their $x$ dependence at some scale $Q_{0}$, the evolution of the FF's with $Q$ may be computed perturbatively from the timelike
Altarelli-Parisi equations, which are presently known through NLO [7]. This method to determine $\alpha_{s}^{(5)}\left(M_{Z}\right)$ is particularly clean in the sense that, unlike other methods based on $e^{+} e^{-}$data, it is not plagued by uncertainties associated with hadronization corrections, jet algorithms, etc. We recall that, similar to the scaling violations in the parton density functions, perturbative QCD predicts only the $Q^{2}$ dependence of the FF's. Therefore, measurements at different $\mathrm{CM}$ energies are needed in order to extract values of $\alpha_{s}^{(5)}\left(M_{Z}\right)$. Furthermore, since the $Q^{2}$ evolution mixes the quark and gluon FF's, it is essential to determine all FF's individually.

In 1994-1995, two of us, together with Binnewies, extracted $\pi^{ \pm}$and $K^{ \pm}$FF's through fits to PEP and partially preliminary LEP1 data and thus determined $\alpha_{s}^{(5)}\left(M_{Z}\right)$ to be $0.118(0.122)$ at NLO (LO) [8] (BKK). However, these analyses suffered from the lack of specific data on the fragmentation of tagged quarks and gluons to $\pi^{ \pm}, K^{ \pm}$, and $p / \bar{p}$ hadrons. This drawback has been cured in 1998 by the advent of a wealth of new data from the LEP1 and SLC experiments [9-13]. The data partly come as light-, $c$-, and $b$-quark-enriched samples with identified final-state hadrons $\left(\pi^{ \pm}, K^{ \pm}\right.$, and $\left.p / \bar{p}\right)$ [9-11] or as gluon-tagged three-jet samples without hadron identification $[12,13]$. This new situation motivates us to update, refine, and extend the BKK analysis [8] by generating new LO and NLO sets of $\pi^{ \pm}, K^{ \pm}$, and $p / \bar{p}$ FF's. By also including in our fits $\pi^{ \pm}, K^{ \pm}$, and $p / \bar{p}$ data (without flavor separation) from PEP [14], with CM energy $\sqrt{s}=29 \mathrm{GeV}$, we obtain a handle on the scaling violations in the FF's, which allows us to extract LO and NLO values of $\alpha_{s}^{(5)}\left(M_{Z}\right)$. The latter data [14] combines small statistical errors with fine binning in $x$ and is more constraining than other data from the pre-LEP1/SLC era.

The NLO formalism for extracting FF's from $e^{+} e^{-}$ data was comprehensively described in Ref. [8]. We work in the $\overline{\mathrm{MS}}$ renormalization and factorization scheme and choose the renormalization scale $\mu$ and the factorization scale $M_{f}$ to be $\mu=M_{f}=\xi \sqrt{s}$, except for gluon-tagged three-jet events, where we put $\mu=M_{f}=2 \xi E_{\text {jet }}$, with $E_{\text {jet }}$ being the gluon jet energy in the CM frame. Here the dimensionless parameter $\xi$ is introduced to determine the 
theoretical uncertainty in $\alpha_{s}^{(5)}\left(M_{Z}\right)$ from scale variations. As usual, we allow for variations between $\xi=1 / 2$ and 2 around the default value 1 . For the actual fitting procedure, we use $x$ bins in the interval $0.1 \leq x \leq 1$ and integrate the theoretical functions over the bin widths as is done in the experimental analyses. The restriction at small $x$ is introduced to exclude events in the region where mass effects and nonperturbative intrinsic-transverse-momentum effects are important and the underlying formalism is insufficient. On the other hand, our analysis should be rather insensitive to nonperturbative effects at $x$ values close to 1 , since the experimental errors are very large there. We parametrize the $x$ dependence of the FF's at the starting scale $Q_{0}$ as $D_{a}^{h}\left(x, Q_{0}^{2}\right)=N x^{\alpha}(1-x)^{\beta}$. We treat $N, \alpha$, and $\beta$ as independent fit parameters. In addition, we take the asymptotic scale parameter $\Lambda \frac{(5)}{\mathrm{MS}}$, appropriate for five quark flavors, as a free parameter. Thus, we have a total of 46 independent fit parameters. The quality of the fit is measured in terms of the $\chi^{2}$ value per degree of freedom, $\chi_{\mathrm{DF}}^{2}$, for all selected data points. Using a multidimensional minimization algorithm [15], we search this 46-dimensional parameter space for the point at which the deviation of the theoretical prediction from the data becomes minimal.

The $\chi_{\mathrm{DF}}^{2}$ values achieved for the various data sets used in our LO and NLO fits may be seen from Table I. Most of the $\chi_{\mathrm{DF}}^{2}$ values lie around unity or below, indicating that

TABLE I. CM energies, types of data, and $\chi_{\mathrm{DF}}^{2}$ values obtained at LO and NLO for the various data samples.

\begin{tabular}{|c|c|c|c|}
\hline$\sqrt{s}[\mathrm{GeV}]$ & Data type & \multicolumn{2}{|c|}{$\chi_{\mathrm{DF}}^{2}$ in $\mathrm{NLO}(\mathrm{LO})$} \\
\hline 29.0 & $\begin{array}{ll}\sigma^{\pi} & (\text { all }) \\
\sigma^{K} & \text { (all) } \\
\sigma^{p} & (\text { all })\end{array}$ & $\begin{array}{l}0.64(0.71)[14] \\
1.86(1.40)[14] \\
0.79(0.70)[14]\end{array}$ & \\
\hline \multirow[t]{11}{*}{91.2} & $\begin{array}{l}\sigma^{h}(\text { all }) \\
\sigma^{h}(u d s) \\
\sigma^{h}(b)\end{array}$ & $\begin{array}{l}1.28(1.40)[10] \\
0.20(0.20)[10] \\
0.43(0.41)[10]\end{array}$ & $1.32(1.44)[11]$ \\
\hline & $\sigma^{\pi}($ all $)$ & $\begin{array}{l}1.28(1.65)[9] \\
0.58(0.60)[10]\end{array}$ & $3.09(3.13)[11]$ \\
\hline & $\begin{array}{ll}\sigma^{\pi} & (u d s) \\
\sigma^{\pi} & (c)\end{array}$ & $0.72(0.73)[10]$ & $\begin{array}{l}1.87(2.17)[11] \\
1.36(1.16)[11]\end{array}$ \\
\hline & $\sigma^{\pi}(b)$ & $0.57(0.58)[10]$ & $1.00(0.99)[11]$ \\
\hline & $\sigma^{K}($ all $)$ & $\begin{array}{l}0.30(0.32)[9] \\
0.86(0.79)[10]\end{array}$ & $0.44(0.45)[11]$ \\
\hline & $\begin{array}{l}\sigma^{K}(u d s) \\
\sigma^{K}(c)\end{array}$ & $0.53(0.60)$ [10] & $\begin{array}{l}0.65(0.64)[11] \\
2.11(1.90)\end{array}$ \\
\hline & $\sigma^{K}(b)$ & $0.14(0.14)[10]$ & $1.21(1.23)[11]$ \\
\hline & $\sigma^{p}($ all $)$ & $\begin{array}{l}0.93(0.80)[9] \\
0.09(0.06)[10]\end{array}$ & $0.79(0.70)[11]$ \\
\hline & $\sigma^{p}(u d s)$ & $0.11(0.14)[10]$ & $1.29(1.28)[11]$ \\
\hline & $\sigma^{p}(c)$ & & $0.92(0.89)[11]$ \\
\hline & $\sigma^{p}(b)$ & $0.56(0.62)[10]$ & $0.97(0.89)[11]$ \\
\hline \multicolumn{4}{|l|}{$E_{\text {jet }}[\mathrm{GeV}]$} \\
\hline 26.2 & $D_{g}^{h}$ & 1.19 (1.18) [12] & \\
\hline 40.1 & $D_{g}^{h}$ & $1.03(0.90)[13]$ & \\
\hline
\end{tabular}

the fitted FF's describe all data sets within their respective errors. In general, the $\chi_{\mathrm{DF}}^{2}$ values come out slightly in favor for the DELPHI [10] data. The overall goodness of the NLO (LO) fit is given by $\chi_{\mathrm{DF}}^{2}=0.98(0.97)$. The goodness of our fit may also be judged from Figs. 1 and 2, where our LO and NLO fit results are compared with the ALEPH [9,12], DELPHI [10], OPAL [13], and SLD [11] data. In Fig. 1, we study the differential cross section $\left(1 / \sigma_{\text {tot }}\right) d \sigma^{h} / d x$ of process (1) for $\pi^{ \pm}, K^{ \pm}, p / \bar{p}$, and unidentified charged hadrons at $\sqrt{s}=91.2 \mathrm{GeV}$, normalized to the total hadronic cross section $\sigma_{\text {tot }}$, as a function of the scaled momentum $x=2 p_{h} / \sqrt{s}$. As in Refs. [10,11], we assume that the sum of the $\pi^{ \pm}, K^{ \pm}$, and $p / \bar{p}$ data exhaust the full charged-hadron data. We observe that, in all cases, the various data are mutually consistent with each other and are nicely described by the LO and NLO fits, which is also reflected in the relatively small $\chi_{\mathrm{DF}}^{2}$ values given in Table I. The LO and NLO fits are almost indistinguishable in those regions of $x$, where the data have small errors. At large $x$, where the statistical errors are large, the LO and NLO results sometimes moderately deviate from each other. In Fig. 2, we compare the ALEPH [12] and OPAL [13] measurements of the

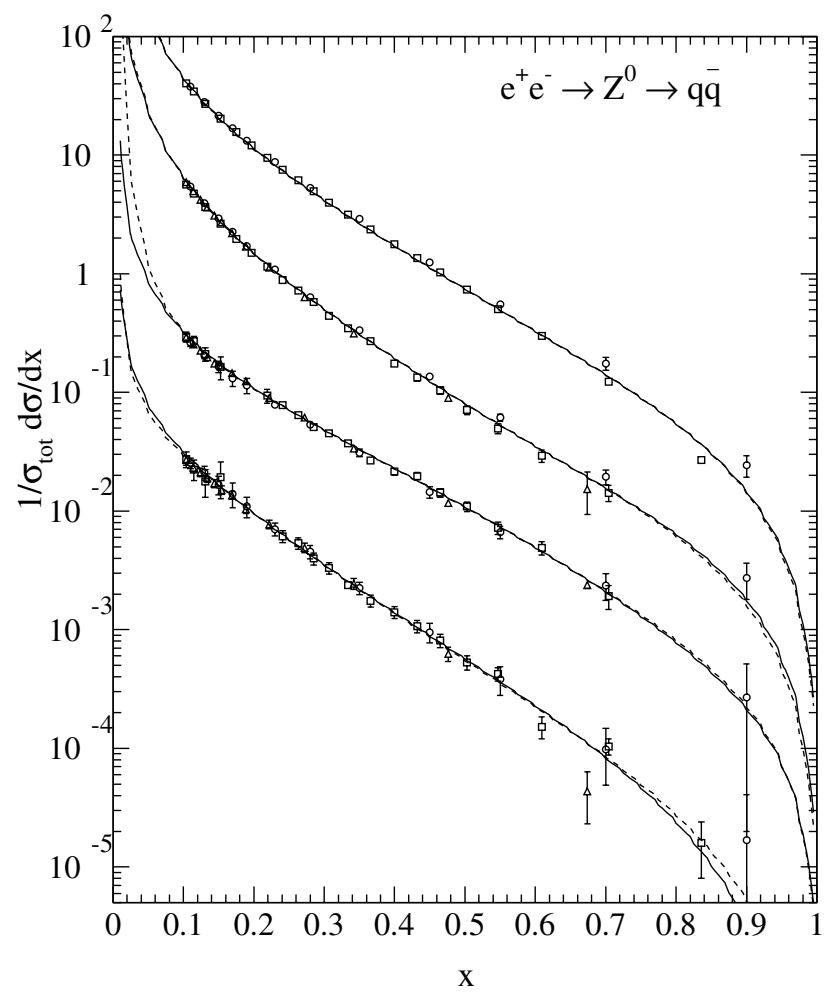

FIG. 1. Normalized differential cross section of inclusive hadron production at $\sqrt{s}=91.2 \mathrm{GeV}$ as a function of $x$. The LO (dashed lines) and NLO (solid lines) fit results are compared with data from ALEPH [9] (triangles), DELPHI [10] (circles), and SLD [11] (squares). The upmost, second, third, and lowest curves refer to charged hadrons, $\pi^{ \pm}, K^{ \pm}$, and $p / \bar{p}$, respectively. Each pair of curves is rescaled relative to the nearest upper one by a factor of $1 / 5$. 


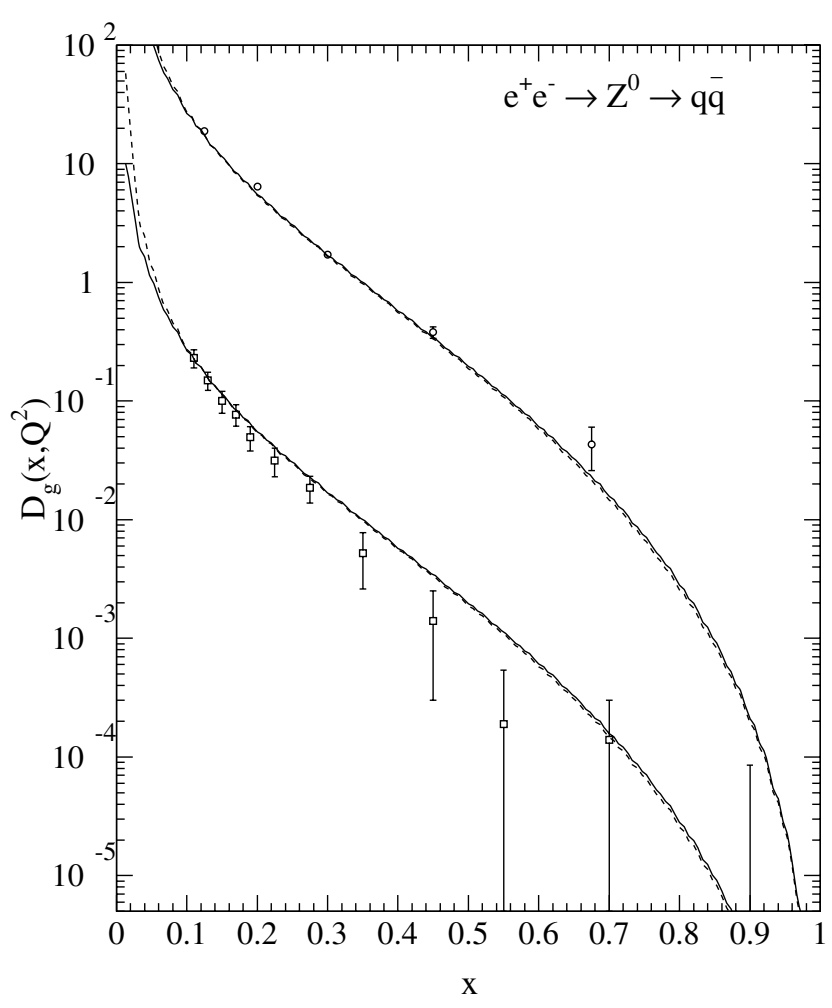

FIG. 2. Gluon FF for charged-hadron production at $Q=52.4$ and $80.2 \mathrm{GeV}$ as a function of $x$. The LO (dashed lines) and NLO (solid lines) predictions are compared with three-jet data from ALEPH [12] with $E_{\text {jet }}=26.2 \mathrm{GeV}$ (upper curves) and from OPAL [13] with $E_{\text {jet }}=40.1 \mathrm{GeV}$ (lower curves). The OPAL data and the pertinent predictions are rescaled by a factor of $1 / 100$.

gluon FF in gluon-tagged charged-hadron production, with $E_{\text {jet }}=26.2$ and $40.1 \mathrm{GeV}$, respectively, with our LO and NLO fit results. The data are nicely fitted, with $\chi_{\mathrm{DF}}^{2}$ values of order unity, as may be seen from Table I. By the same token, this implies that the data $[12,13]$ are mutually consistent.

At low energies, the logarithmic scaling violations in the FF's might receive nonperturbative power-law corrections proportional to $1 / \sqrt{s}$, which effectively displace the perturbative value of $x$ with respect to the observed value $x^{\prime}$ according to $x=x^{\prime}+h_{0}\left(1 / \sqrt{s}-1 / \sqrt{s_{0}}\right)$, where $\sqrt{s_{0}}$ is the scale at which all nonperturbative effects are assumed to be absorbed in the FF's [16]. Incorporating this additional freedom in our NLO (LO) fit for the central scale choice $\xi=1$ and identifying $\sqrt{s_{0}}$ with the lowest CM energy of the selected data samples, $29 \mathrm{GeV}$ [14], we find $h_{0}=(-0.01 \pm 0.10) \mathrm{GeV}\left[h_{0}=\right.$ $(-0.01 \pm 0.11) \mathrm{GeV}]$, which is compatible with zero, indicating that nonperturbative effects are negligible. Similar conclusions were reached by ALEPH [17], who obtained $h_{0}=(-0.14 \pm 0.11) \mathrm{GeV}$ for $\sqrt{s_{0}}=22 \mathrm{GeV}$, and more recently by DELPHI [18], who found $h_{0}=$ $(-0.07 \pm 0.11) \mathrm{GeV}$ for $\sqrt{s_{0}}=14 \mathrm{GeV}$ including also the ALEPH [17] data in their global fit.
The purpose of this Letter is to update and improve the determinations of $\Lambda_{\overline{\mathrm{MS}}}^{(5)}$ and $\alpha_{s}^{(5)}\left(M_{Z}\right)$ from the scaling violations in the FF's. We obtain $\Lambda \frac{(5)}{\mathrm{MS}}=88_{-31}^{+34}{ }_{-23}^{+3} \mathrm{MeV}$ at $\mathrm{LO}$ and $\Lambda \frac{(5)}{\mathrm{MS}}=213_{-73}^{+75}{ }_{-29}^{+22} \mathrm{MeV}$ at NLO, where the first errors are experimental and the second ones are theoretical. The experimental errors are determined by varying $\Lambda \frac{(5)}{\mathrm{MS}}$ in such a way that the total $\chi_{\mathrm{DF}}^{2}$ value is increased by one unit if all the other fit parameters are kept fixed, while the theoretical errors are obtained by repeating the LO and NLO fits for the scale choices $\xi=1 / 2$ and 2 . From the LO and NLO formulas for $\alpha_{s}^{\left(n_{f}\right)}(\mu)$, we thus obtain

$$
\begin{aligned}
& \alpha_{s}^{(5)}\left(M_{Z}\right)=0.1181_{-0.0069}^{+0.0058}+0.0049 \quad(\mathrm{LO}),
\end{aligned}
$$

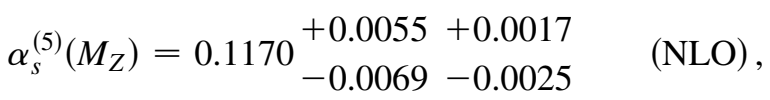

respectively. As expected, the theoretical error is significantly reduced as we pass from LO to NLO. Adding the maximum experimental and theoretical deviations from the central values in quadrature, we find $\Lambda \frac{(5)}{\mathrm{MS}}=(88 \pm$ 41) $\mathrm{MeV}$ and $\alpha_{s}^{(5)}\left(M_{Z}\right)=0.1181 \pm 0.0085$ at $\mathrm{LO}$ and $\Lambda \frac{(5)}{\mathrm{MS}}=(213 \pm 80) \mathrm{MeV} \quad$ and $\quad \alpha_{s}^{(5)}\left(M_{Z}\right)=0.1170 \pm$ 0.0073 at NLO. We observe that our LO and NLO values of $\alpha_{s}^{(5)}\left(M_{Z}\right)$ are quite consistent with each other, which indicates that our analysis is perturbatively stable. The fact that the respective values of $\Lambda \frac{(5)}{\mathrm{MS}}$ significantly differ is a well-known feature of the $\overline{\mathrm{MS}}$ definition of $\alpha_{s}^{\left(n_{f}\right)}(\mu)$ [3].

Our values of $\Lambda \frac{(5)}{\mathrm{MS}}$ and $\alpha_{s}^{(5)}\left(M_{Z}\right)$ agree perfectly with those presently quoted by the Particle Data Group (PDG) [1] as world averages, $\Lambda \frac{(5)}{\mathrm{MS}}=208_{-23}^{+25} \mathrm{MeV}$ and $\alpha_{s}^{(5)}\left(M_{Z}\right)=0.1181 \pm 0.002$, respectively. Notice that, in contrast to our LO and NLO analyses, the PDG evaluates $\Lambda \frac{(5)}{\mathrm{MS}}$ from $\alpha_{s}^{(5)}\left(M_{Z}\right)$ using the three-loop relationship [3]. The PDG combines 12 different kinds of $\alpha_{s}^{(5)}\left(M_{Z}\right)$ measurements, including one from the scaling violations in the FF's $[17,18]$, by minimizing the total $\chi^{2}$ value and thus obtains $\alpha_{s}^{(5)}\left(M_{Z}\right)=0.1181 \pm 0.0014$ with $\chi^{2}=3.8$. The world average cited above is then estimated from the outcome by allowing for correlations between certain systematic errors. It is interesting to investigate how the world average of $\alpha_{s}^{(5)}\left(M_{Z}\right)$ is affected by our analysis. If we replace the value $\alpha_{s}^{(5)}\left(M_{Z}\right)=0.125 \pm 0.005 \pm 0.008$ resulting from previous FF analyses [17,18], which enters the PDG average, with our new NLO value, then we obtain $\alpha_{s}^{(5)}\left(M_{Z}\right)=0.1180 \pm 0.0014$ with $\chi^{2}=3.22$, i.e., the face value of the world average essentially goes unchanged, while the overall agreement is appreciably improved. This is also evident from the comparison of Fig. 3, which summarizes our updated world average, with the corresponding Fig. 9.1 in Ref. [1]. We observe that the central value of our new NLO result for $\alpha_{s}^{(5)}\left(M_{Z}\right)$ falls into the shaded band, which indicates the error of the world 


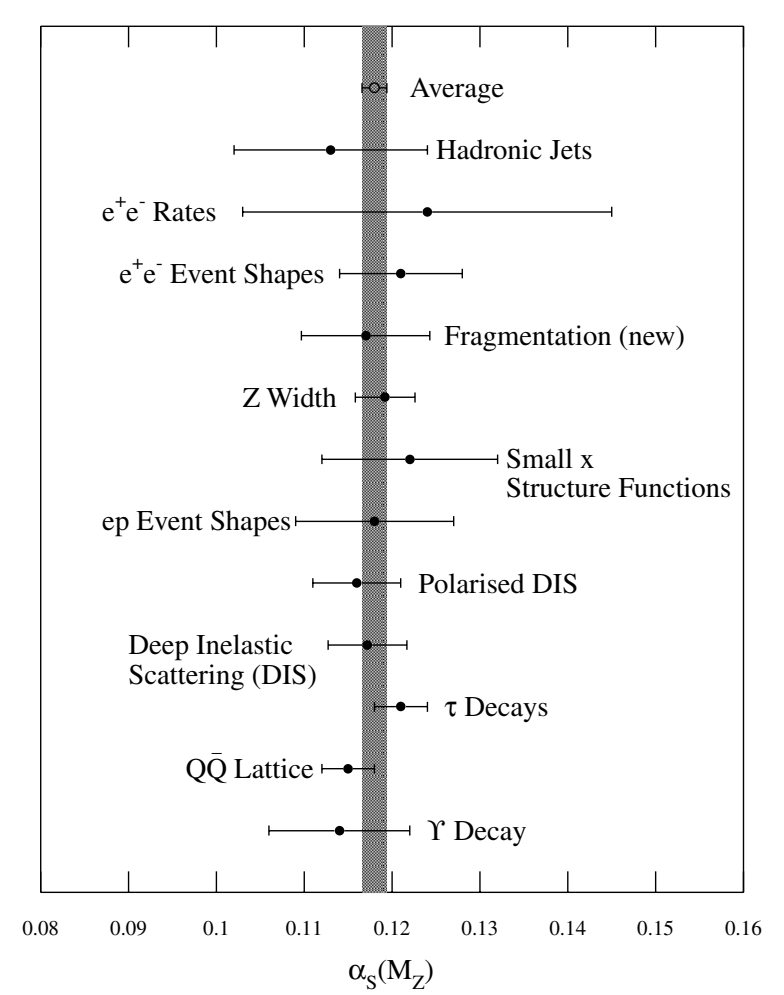

FIG. 3. Summary of the values of $\alpha_{s}^{(5)}\left(M_{Z}\right)$ from various processes. The errors shown represent the total errors including theoretical uncertainties.

average, while in Fig. 9.1 of Ref. [1] the corresponding central value $[17,18]$ exceeds the world average by 3.5 standard deviations of the latter, which is more than for all other 11 processes. Furthermore, our new NLO result has a somewhat smaller error (0.0073) than the corresponding result $[17,18]$ used by the PDG $(0.009)$. This is due to a marked decrease in the theoretical error, which may be attributed to a different choice of input data, especially at low $\mathrm{CM}$ energies. If we take the point of view that our new NLO value of $\alpha_{s}^{(5)}\left(M_{Z}\right)$ should rather be combined with the result from the previous FF analyses [17,18] before taking the world average, then the latter turns out to be $\alpha_{s}^{(5)}\left(M_{Z}\right)=0.1181 \pm 0.0014$ with $\chi^{2}=3.34$.

In summary, we presented an updated and improved determination of $\alpha_{s}^{(5)}\left(M_{Z}\right)$ from the LO and NLO analyses of inclusive light-hadron production in $e^{+} e^{-}$annihilation. Our strategy was to include in our fits only high-precision LEP1 and SLC data with both flavor separation and hadron identification (namely, light-, $c$-, and $b$-quark-enriched samples of $\pi^{ \pm}, K^{ \pm}$, and $p \bar{p}$ data) [10,11], gluon-tagged three-jet samples with a fixed gluon-jet energy $[12,13]$, and the $\pi^{ \pm}, K^{ \pm}$, and $p / \bar{p}$ data sets from the pre-LEP1/SLC era with the highest statistics and the finest binning in $x$ [14]. Our LO and NLO results for $\alpha_{s}^{(5)}\left(M_{Z}\right)$ are given in
Eq. (2). Their central values comfortably lie within the error band of the world average quoted in Ref. [1]. This represents a significant improvement of the overall agreement, since the central value of a previous result from scaling violations in FF's [17,18], which is used in Ref. [1], lies 3.5 standard deviations outside this error band.

This work was supported in part by DFG Grant No. KN 365/1-1, by BMBF Grant No. 05 HT9GUA 3, and by the European Commission through TMR Network No. ERBFMRX-CT98-0194.

[1] Particle Data Group, D. E. Groom et al., Eur. Phys. J. C 15, 1 (2000).

[2] T. van Ritbergen, J.A. M. Vermaseren, and S. A. Larin, Phys. Lett. B 400, 379 (1997).

[3] K. G. Chetyrkin, B. A. Kniehl, and M. Steinhauser, Phys. Rev. Lett. 79, 2184 (1997), and references therein.

[4] S. Bethke, in The Proceedings of the Fourth International Symposium on Radiative Corrections (RADCOR 98): Application of Quantum Field Theory to Phenomenology, Barcelona, Spain, 1998, edited by J. Solà (World Scientific, Singapore, 1999), p. 243.

[5] G. Altarelli, R. K. Ellis, G. Martinelli, and S.-Y. Pi, Nucl. Phys. B160, 301 (1979); R. Baier and K. Fey, Z. Phys. C 2, 339 (1979).

[6] P. J. Rijken and W. L. van Neerven, Phys. Lett. B 386, 422 (1996); 392, 207 (1997); Nucl. Phys. B487, 233 (1997).

[7] G. Curci, W. Furmanski, and R. Petronzio, Nucl. Phys. B175, 27 (1980); W. Furmanski and R. Petronzio, Phys. Lett. 97B, 437 (1980).

[8] J. Binnewies, B. A. Kniehl, and G. Kramer, Z. Phys. C 65 , 471 (1995); Phys. Rev. D 52, 4947 (1995).

[9] ALEPH Collaboration, D. Buskulic et al., Z. Phys. C 66, 355 (1995).

[10] DELPHI Collaboration, P. Abreu et al., Eur. Phys. J. C 5, 585 (1998).

[11] SLD Collaboration, K. Abe et al., Phys. Rev. D 59, 052001 (1999).

[12] ALEPH Collaboration, R. Barate et al., Eur. Phys. J. C 17, 1 (2000).

[13] OPAL Collaboration, G. Abbiendi et al., Eur. Phys. J. C 11, 217 (1999).

[14] TPC/Two-Gamma Collaboration, H. Aihara et al., LBL Report No. LBL-23737, UC-34D, 1988 (unpublished); Phys. Rev. Lett. 61, 1263 (1988).

[15] F. James and M. Roos, Comput. Phys. Commun. 10, 343 (1975).

[16] P. Nason and B. R. Webber, Nucl. Phys. B421, 473 (1994); B480, 755(E) (1996).

[17] ALEPH Collaboration, D. Buskulic et al., Phys. Lett. B 357, 487 (1995); 364, 247(E) (1995).

[18] DELPHI Collaboration, P. Abreu et al., Phys. Lett. B 398, 194 (1997). 